\title{
EXPERIMENTAL INVESTIGATION OF THERMALLY INDUCED INTERACTING CRACKS IN BRITTLE SOLIDS
}

\author{
John F. GeYer $\dagger$ and S. Nemat-Nasser \\ The Technological Institute, Northwestern University, Evanston, IL 60201, U.S.A.
}

(Received 4 September 1980; in revised form 23 January 1981)

\begin{abstract}
The results of an experimental investigation of thermally induced parallel edge cracks in a half-plane consisting of brittle material are reported. A free edge of a glass plate heated to a uniform temperature, was brought in contact with a liquid bath cooled by dry ice. The thermal contraction of the boundary layer produced interacting tension cracks. When no initial cracks existed, a large amount of potential energy was released in the formation of a few cracks which extended in a dynamic manner beyond the thermal layer. To prevent this type of cracking, initial cracks were formed before thermally inducing crack growth. This resulted in stable crack growth which qualitatively agreed well with the previously developed theory. The quantitative comparison was also reasonably good.
\end{abstract}

\section{INTRODUCTION}

In a series of papers, Nemat-Nasser[1] and Nemat-Nasser, Keer et al.[2-5] have studied the growth pattern of a system of interacting parallel edge cracks induced in a linear elastic brittle half-space by the formation of a thermal layer at the free surface. The motivation for these studies relates to the process of extracting heat from hot dry rock masses, a project which recently has been initiated and implemented at the Los Alamos Scientific Laboratory in New Mexico. For further discussion of this geothermal energy extraction process and additional applications, see Nemat-Nasser [5], and Nemat-Nasser et al.[2].

The purpose of this experimental study was to qualitatively and quantitatively verify the theoretical results of the previous studies. Glass plates were heated to a uniform temperature and then brought in contact with a liquid bath cooled by dry ice. Because of the cooling of the contacting edge, a thermal boundary layer develops in the solid. The thermal contraction of the boundary layer produces tension cracks which propagate perpendicular to the cooled edge. As the thermal layer increases the cracks continue to grow. Previous theoretical studies have shown that when the crack spacing is large compared to their lengths, their interaction is weak and therefore they grow at equal rates. However, when the crack spacing and length are of the same order of magnitude, the crack growth becomes highly interactive. When a critical state is reached alternate cracks stop, while the others continue to grow at a faster rate. This process continues until another critical state is reached, where the cracks that had stopped might suddenly close depending on the temperature profile, as the others suddenly jump into a finitely longer length.

Initially it was not considered necessary to precrack the glass plates at the cooling edge. However, the results from this first set of experiments appeared unsatisfactory: a few cracks extended far beyond the thermal layer. The cracking occurred in a dynamic fashion seconds after initial contact with the dry ice bath. In an attempt to produce stable growth, notches were ground along the free edge with a sharp tip at the end of the notch. Despite this preparation, dynamic cracking still occurred. Finally, initial cracks were created at constant spacing along the free edge by scratching the plate with a glass cutter and then tapping the crack open. These plates heated to a slightly lower temperature produced stable crack growth in agreement with the theoretical predictions. The first set of experiments, however, showed a different and interesting phenomena: dynamic crack growth which relates more to the problem of "crack initiation" rather than "crack growth". 


\section{EXPERIMENTAL APPARATUS}

Plates of glass were suspended inside the insulated heating package as shown in Fig. 1. Nickel-chromium wire connected to a variable power source and attached directly to the insulation sheets provided the heating source. There were three separate heating wires: two located on the front and back insulation panels, and the third attached to the removable bottom panel. The heaters were capable of producing temperatures as high as $200^{\circ} \mathrm{C}$ with the given power source. The dimensions inside the package were $50 \mathrm{~cm}$ by $50 \mathrm{~cm}$ by $5.4 \mathrm{~cm}$ deep, with the glass $47 \mathrm{~cm}$ wide by $42 \mathrm{~cm}$ high. The glass plate was suspended from above by wire and hooks which could be lowered and raised. After hanging and preparing the glass plate, the front insulation panel was clamped in place. The bottom heater was then held in position with wedges to form the enclosed heating package.

To monitor the temperature of the glass, thermocouples were attached at specified locations using silicone adhesive. The thermocouples were connected to a potentiometer with a strip gauge recorder, as pictured in Fig. 1, to continuously chart the temperature. Typically eight thermocouples were spaced at $1.3 \mathrm{~cm}$ intervals to record the vertical thermal gradient. In order to achieve a uniform temperature distribution in the glass, two sheets of $0.6 \mathrm{~cm}$ thick aluminum were hung on either side of the glass. The rear aluminum plate can be seen behind the glass in Fig. 1. With the aluminum plates and with the heating wires extended over the entire height of glass, the temperature varied by only about $5^{\circ} \mathrm{C}$ from top to bottom and about $2^{\circ} \mathrm{C}$ from edge to center.

The cooling bath consisted of an aluminum channel held in a styrofoam container. Blocks of dry ice were kept in contact with the channel by sprung steel plates. Silicone liquid was then poured onto the channel to provide the cooling medium in uniform contact with the heated glass plate. This liquid was selected for its stable liquid properties under the operating temperatures. The temperature of the liquid was measured to within $1^{\circ} \mathrm{C}$ of dry ice temperature $\left(-78^{\circ} \mathrm{C}\right)$. After heating the glass plate to the desired temperature, the bottom heater was removed and the dry ice bath was positioned under the heating package. When the cooling bath was raised $1 \mathrm{~cm}$, foam padding attached to the top of the styrofoam box prevented air circulation around the glass.

\section{EXPERIMENTAL PROCEDURE AND RESULTS}

The first set of experiments performed were with $0.6 \mathrm{~cm}$ thick glass plates heated to about $185^{\circ} \mathrm{C}$. These plates had no special preparation, i.e. no initial crack existed. A typical result is shown in Fig. 2. The cracks have been highlighted with a felt pen to show the crack regime

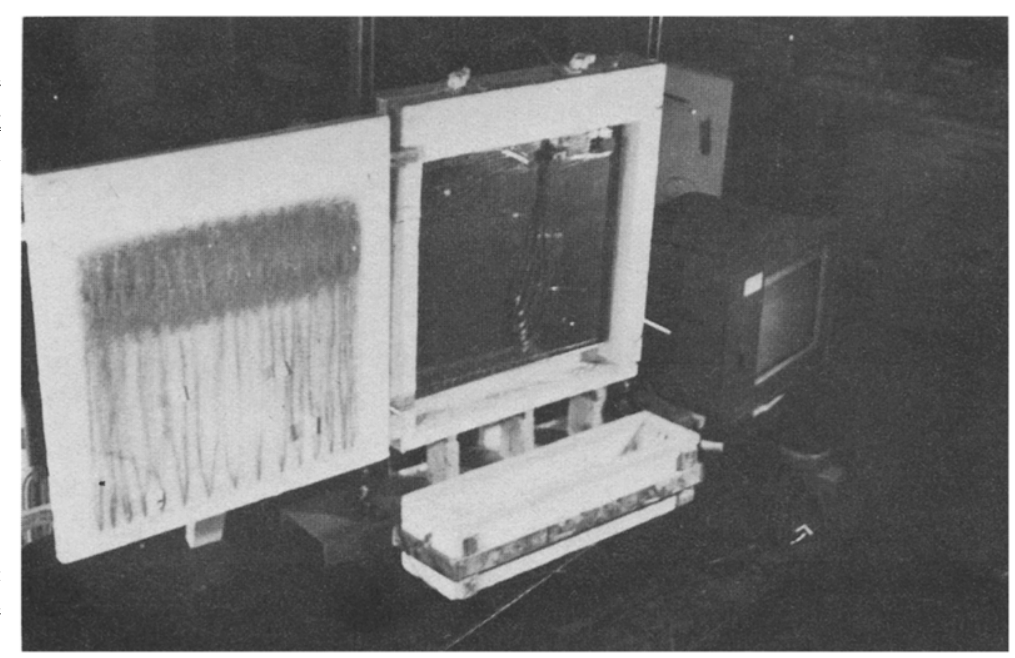

Fig. 1. Overview of heating package with front panel opened and shown with cooling bath (below) and potentiometer (to the right). 
more clearly. A few cracks have extended $25 \mathrm{~cm}$ or more, which is far beyond the thermal zone and into the uniformly heated no-tension region. Another test was performed with the heating panel and aluminum plate removed so the crack growth could be observed. Seconds after the plate came in contact with the cooled bath, a few cracks "shot" up as much as $20 \mathrm{~cm}$. This quasi-dynamic growth was not time dependent or controlled, contrary to the theoretical assumptions. A large amount of potential energy stored in the plate was released with the growth of the few cracks. The other cracks formed later as the (cooled) thermal zone increased. These grew in a stable fashion and the overall pattern was in agreement with the theory. The cracks on the right side of Fig. 2 in particular behaved as predicted.

Plots of the temperature profile of the plate while cooling are similar to the profile of error function given by Nemat-Nasser et al.[2] to account for heat conduction; see Fig. 3. For this temperature profile, unlike that for the profile which accounts for the convective heat exchange, no crack closure takes place. Cracks grow until a critical state is reached, then alternate cracks stop, while the remaining ones grow at a faster rate. When the next critical state is reached every other of those cracks which had continued growing stops, while the remaining ones continue to grow at a faster rate. This process repeats itself as the depth of the thermal layer increases. This was the general pattern pictured here. Cracks stopped at certain levels, with alternate cracks usually longer. Even the long cracks fit this interacting pattern to some extent, although the theory does not apply to the type of growth which was involved. The cracks were

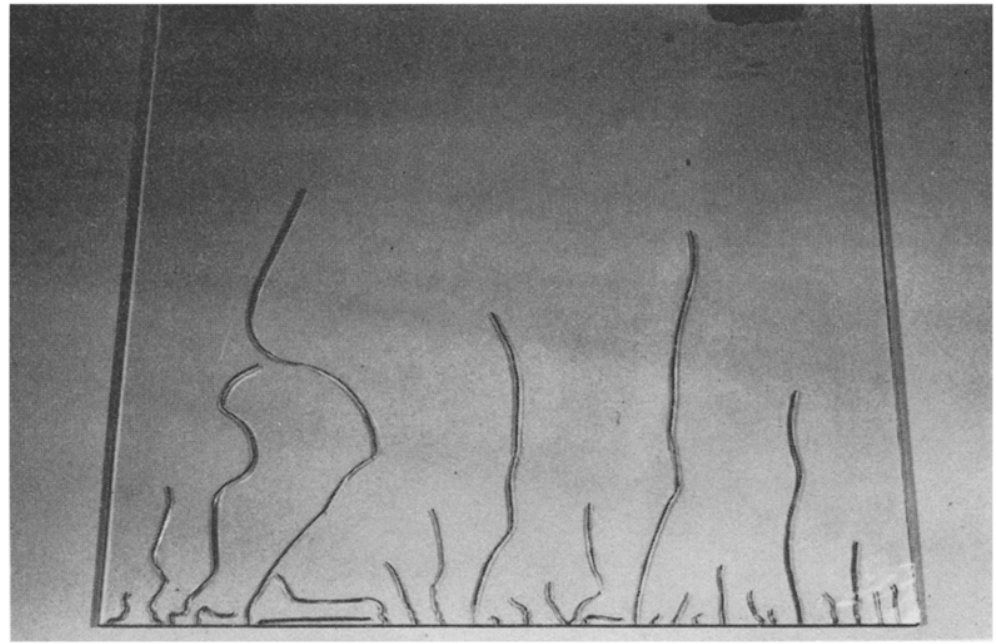

Fig. 2. Crack regime for plate with no initial cracks.

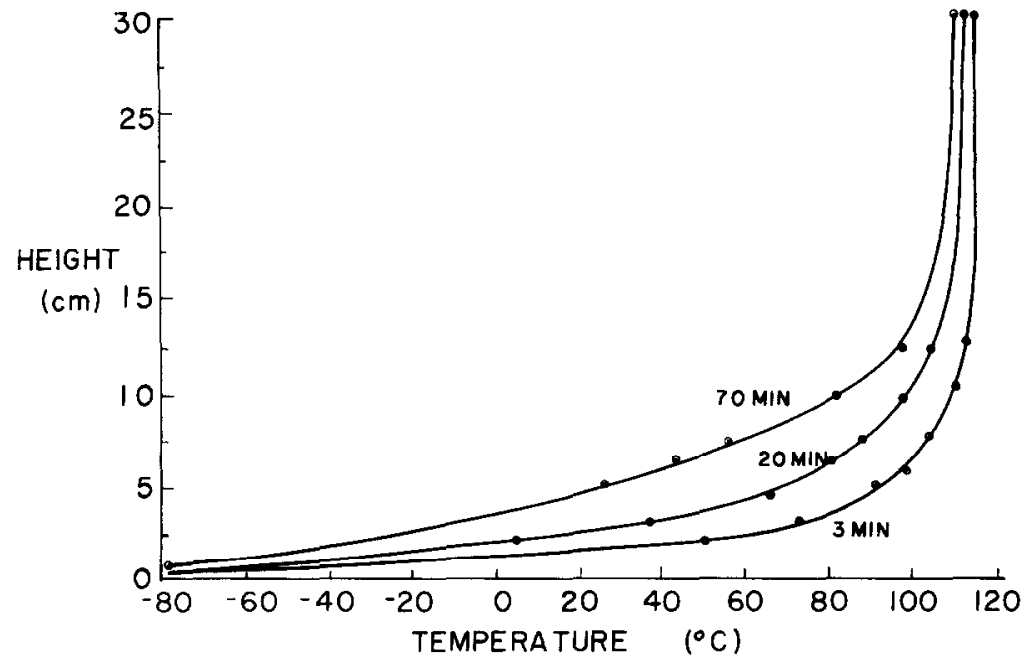

Fig. 3. Temperature profile at three successive times after contact with cooling bath for plate shown in Fig. 5. 
not all straight and perpendicular to the free edge; crack curving occurred also. This could be on account of the residual stresses $\dagger$ and the nonuniform temperature distribution in the plate. The initial crack spacing was nearly uniform except in the regions where the cracks curved and ran parallel with the free edge. The average spacing was about $1.0 \mathrm{~cm}$. Another interesting result was the nearly constant total crack length for an initial temperature level. Adding the length of each crack for the five tests run at $185^{\circ} \mathrm{C}$, the average length was $159 \mathrm{~cm}$ to within $4 \mathrm{~cm}$ : an input of a certain amount of energy created a constant amount of cracking.

For the next series of experiments the free edge of the glass plates was notched at regular intervals. A grinding wheel was used to produce $0.16 \mathrm{~cm}$ wide notches. A sharp tip was ground at the end of the notch with a diamond powder coated steel plate. It was thought this would allow the plate to contract initially without dynamic cracking. The notches were spaced at $1.3 \mathrm{~cm}$ intervals with a depth of $1.3 \mathrm{~cm}$ using $0.6 \mathrm{~cm}$ thick plate glass. A representative crack regime for this plate (heated to $185^{\circ} \mathrm{C}$ ) is pictured in Fig. 4. Dynamic cracking has again occurred. Loud clicks could be heard after about one or two minutes, as these cracks extended beyond the thermal layer. Other cracks formed in a stable fashion, growing as the thermal layer extended. All cracks initiated at the tip of the notch, with a typical spacing of every third notch. The total crack length was surprisingly close to that of the first procedure, with an average length of $157 \mathrm{~cm}$. Another notched plate was tested at a lower temperature, $130^{\circ} \mathrm{C}$, with dynamic cracking still occurring.

A third experimental procedure resulted in a much more stable growth pattern. Initial cracks were created along the free edge by scratching the glass with a glass cutter and then tapping the cracks open. Thinner plate glass was used, either single strength glass $(0.25 \mathrm{~cm}$ thick) or double strength glass $(0.33 \mathrm{~cm}$ thick). For the plate shown in Fig. 5 single strength glass was used with an initial crack spacing of $1.3 \mathrm{~cm}$ and length $1.8 \mathrm{~cm}$ heated to $115^{\circ} \mathrm{C}$. After cooling the glass at one edge for an hour, a nearly steady state temperature condition resulted since the two main heaters were left on, see Fig. 3. The thermal layer had stopped increasing. At this point the front heating panel and aluminum plate were removed and the crack lengths marked. Some cracks extended further while the plate came to room temperature and on being handled, however the crack lengths were highlighted with a pen for the steady state conditions. The crossed portion of the cracks in Fig. 5 occurred after steady state conditions and because of handling. The lower mark on the cracks indicated the initial crack lengths. Four levels of crack growth existed for the cracks shown in Fig. 5 with average heights of $1.8 \mathrm{~cm}, 3.3 \mathrm{~cm}, 6.4 \mathrm{~cm}$ and $13.7 \mathrm{~cm}$. The basic pattern of alternate cracks stopping and propagating was displayed clearly here. Only the longer cracks had appreciable curving, with this occurring as they reached their final height where the interaction between cracks was weaker. The glass plate pictured in Fig. 6 ,

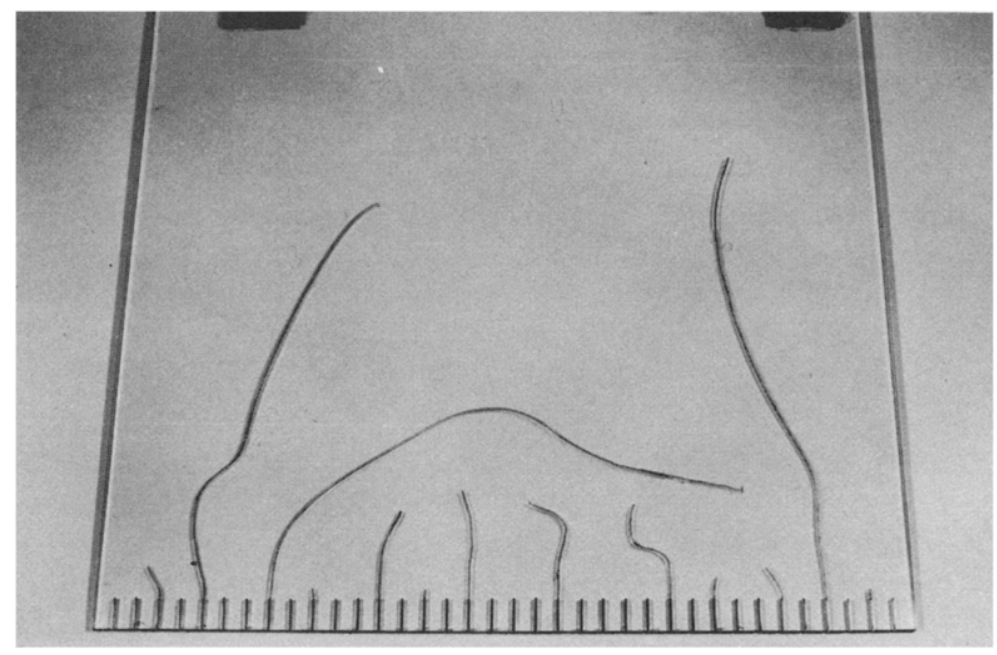

Fig. 4. Crack regime for notched plate. 


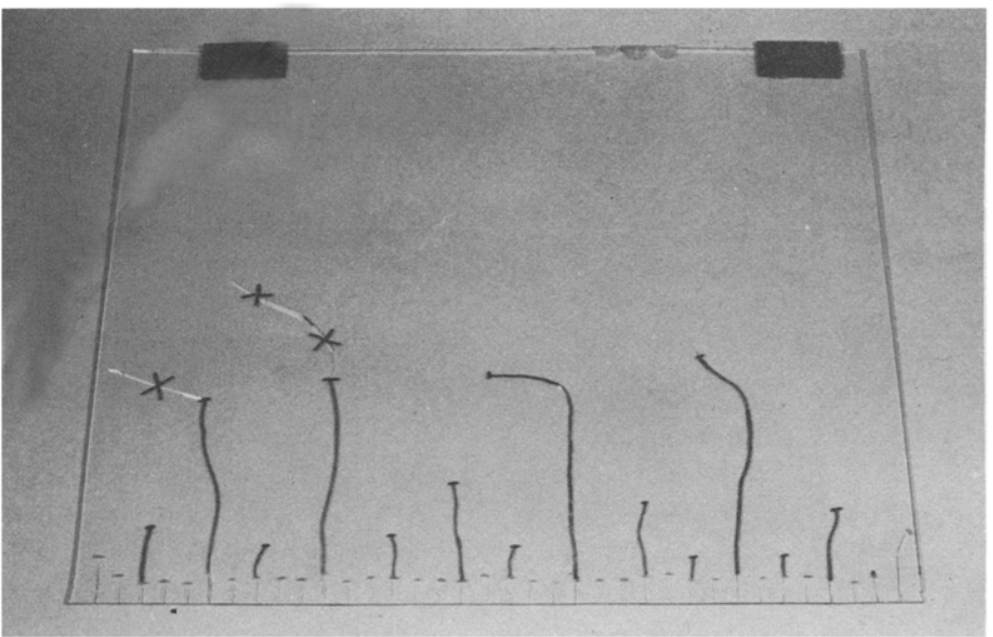

Fig. 5. Crack regime for plate with initial cracks; crack ends are marked at the end of the experiment and further crack extension is denoted by crosses.

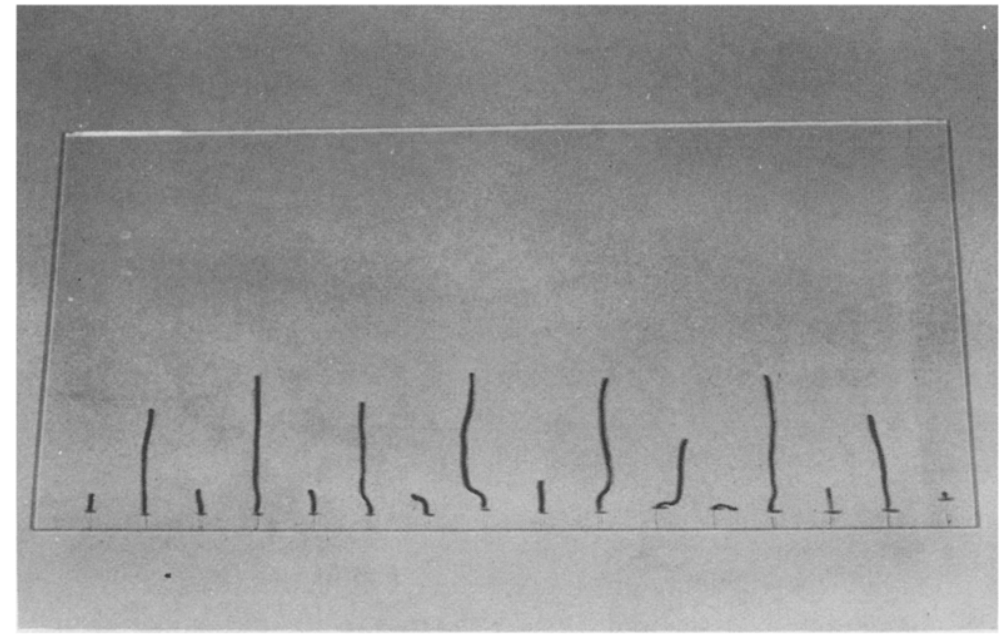

Fig. 6. Crack regime for initially cracked plate tested at room temperature.

was tested at room temperature. Initial cracks spaced at $2.5 \mathrm{~cm}$ and $0.7 \mathrm{~cm}$ deep all became active while undergoing stable growth. The crack lengths for each of the three layers were consistent. Figure 7 presents a distinct picture of near ideal behavior. This single strength glass was heated to $52^{\circ} \mathrm{C}$ with initial cracks $1.3 \mathrm{~cm}$ long spaced at $2.5 \mathrm{~cm}$. All cracks became active while growing stably to three crack levels.

The qualitative pattern displayed in these experiments agrees well with the theory.

\section{CALCULATIONS AND QUANTITATIVE COMPARISONS}

The quantitative results compare reasonably well with the theoretical values. The following glass properties are used for the calculations: $E=6.9 \times 10^{10} \mathrm{~N} / \mathrm{m}^{2}, \nu=0.23$, coefficient of thermal expansion $\hat{\alpha}=8.5 \times 10^{-6} /{ }^{\circ} \mathrm{C}$, critical stress intensity factor $K_{c}=0.5 \mathrm{MN} / \mathrm{m}^{3 / 2}$, and surface energy $\gamma=1.8 \mathrm{~N} / \mathrm{m}$.

The minimum crack spacing can be calculated by an energy consideration[4], which for plane stress leads to

$$
b \geq \frac{2 \gamma}{\hat{\alpha}^{2} E K_{0} T_{0}^{2}}
$$

where $K_{0}$ is a numerical parameter depending on the temperature profile. Using $K_{0}=0.1$ for 


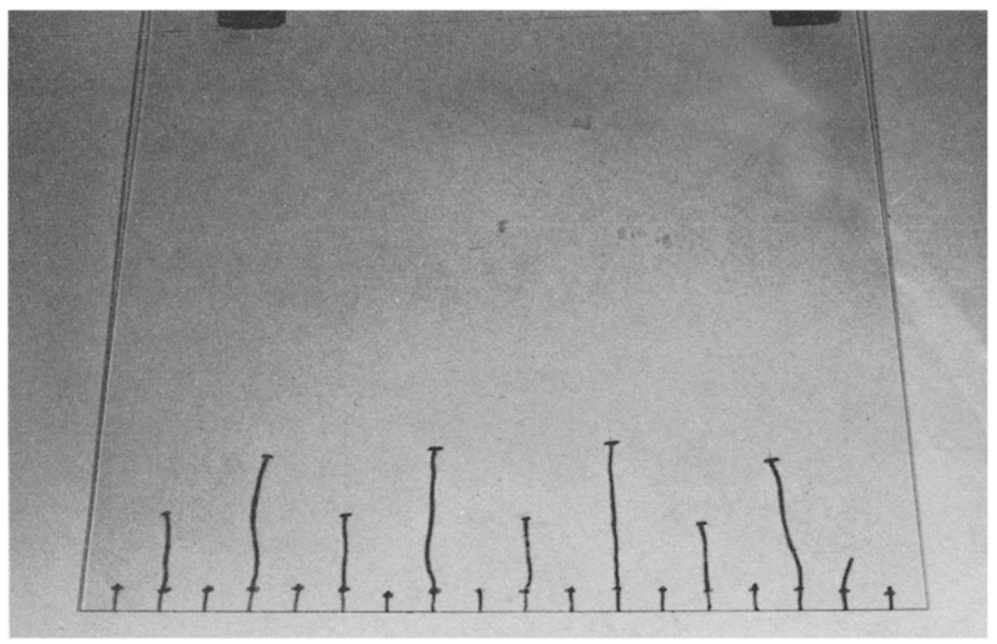

Fig. 7. Crack regime for plate with initial cracks; crack ends are marked at the end of the experiment.

involved temperature profile and $T_{0}=263^{\circ} \mathrm{C}$ we obtain $b=0.010 \mathrm{~cm}$. For the first set of tests with no initial cracks, a spacing of $1.0 \mathrm{~cm}$ is observed. This indicates that only a small portion of the energy available to generate new crack surfaces was thus used. The surplus energy available could explain why a few cracks extend dynamically far into the no-tension zone.

The theoretical crack lengths for the plate tested at room temperature (shown in Fig. 6), will next be calculated. The dimensionless quantities are: $\Delta=\delta / b, a=h / b, N=K_{c} /\left(\sqrt{ }(2 \pi) \hat{\beta} T_{0} \vee b\right)$, where $\delta=$ thermal depth, $b=$ crack spacing, $h=$ crack length and $\hat{\beta}=\hat{\alpha} E$. The initial dimensionless stress intensity factor for $b=2.5 \mathrm{~cm}$ and $T_{0}=100^{\circ} \mathrm{C}$ is $N=0.021$. From the graphs found in [7], (see Appendix A), the critical $N=0.021$ corresponds to $\Delta=1.4$ and $a=1.2$. Since the initial crack length $h=0.8 \mathrm{~cm}$ yields $a=0.3$ which falls below the critical $a=1.2$, all the cracks should be active and grow to a length of $h=3.0 \mathrm{~cm}$ before stopping. Then alternate cracks remain stationary while others grow with a spacing of $5.0 \mathrm{~cm}$. The critical $N$ then is $0.021 / \sqrt{ } 2=0.015$, due to the doubling of the crack spacing. This new value of $N$ relates to $\Delta=1.6$ and $a=1.4$. Thus the second level of cracks should be at a length $h=(1.4)(5.0 \mathrm{~cm})=$ $7.0 \mathrm{~cm}$. Proceeding likewise the length of the third level would be $17.6 \mathrm{~cm}$. The actual lengths of the three levels of cracks average to $1.8 \mathrm{~cm}, 5.8 \mathrm{~cm}$ and $7.1 \mathrm{~cm}$, compared to the theoretical $3.0 \mathrm{~cm}, 7.0 \mathrm{~cm}$ and $17.6 \mathrm{~cm}$. The length of the third level is much lower than the theoretical value because these cracks lack enough thermal driving force to extend to a length where they would interact. The other two values do not correlate exactly for many reasons. The material properties used are only estimates for glass and could differ from the particular values for these plates. The theoretical calculations are based on an assumed error function temperature distribution which probably does not agree exactly with the actual profile, particularly for the first crack level. Had the initial imperfections in crack spacing been accounted for, the theoretical crack lengths would decrease and be closer to the actual values. Considering the assumptions in the theory and other properties, the quantitative correlation was not too bad. Qualitatively the results looked very good.

When these calculations are applied to the heated plates the comparison is similar. For example the cracks pictured in Fig. 5 are examined. The initial dimensionless stress intensity factor for $b=1.3 \mathrm{~cm}$ and $T_{0}=200^{\circ} \mathrm{C}$ is $N=0.016$, which corresponds to $\Delta=1.5$ and $a=1.3$. The actual value of $a=1.4$ lies above this critical value so the first level of cracks would not become active. The predicted length of the first level is $h=(1.3)(1.3 \mathrm{~cm})=1.7 \mathrm{~cm}$ at which alternate cracks stop. The next set of cracks should have grown until $N=0.016 / \sqrt{ } 2=0.011$ or $a=1.6$ or $h=(1.6)(2.5 \mathrm{~cm})=4.0 \mathrm{~cm}$. Similarly the third level should have stopped at a length of $9.7 \mathrm{~cm}$, while the fourth level at $25.1 \mathrm{~cm}$. The observed levels averaged to $1.8 \mathrm{~cm}, 3.3 \mathrm{~cm}, 6.4 \mathrm{~cm}$ and $13.7 \mathrm{~cm}$, compared to the predicted $1.7 \mathrm{~cm}, 4.0 \mathrm{~cm}, 9.7 \mathrm{~cm}$ and $25.1 \mathrm{~cm}$. The fourth layer as before did not reach its final length of interaction so these values should not be compared. The theory predicts every other crack should be active, which compares well with the observed 
result of every second or third crack extending. The quantitative comparison for this plate is again reasonably accurate.

In a recent work Oranratnachai et al. [8] have examined the effect of unequal initial crack spacing on the stability of the thermally induced crack growth regime in brittle solids. These authors show that if the initial spacing is unequal by about $10 \%$, then the crack length at which every third crack ceases to grow may be reduced by more than $30 \%$. If the above estimated crack lengths are reduced by $20-30 \%$, then very good correlation with observed results would be obtained. From the crack patterns shown in Figs. 5-7, it is clear that 5-10\% geometric imperfection should be expected.

Acknowledgements-This work was supported in part by National Science Foundation Grant No. ENG77-22155 to Northwestern University and in part by Northwestern University, partially by a Cabell Fellowship for graduate study (J.F.G.). Appreciation is extended to Mr. John Schmidt for help and advice concerning the experimental apparatus and procedure.

\section{REFERENCES}

1. S. Nemat-Nasser, Stability of a system of interacting cracks. Lett. in Appl. Engng and Sci. 16, 277-285 (1978).

2. S. Nemat-Nasser, L. M. Keer and K. S. Parihar, Unstable growth of thermally induced interacting cracks in brittle solids. Int. J. Solids Structures 14, $409-430$ (1978).

3. L. M. Keer, S. Nemat-Nasser and A. Oranratnachai, Unstable growth of thermally induced interacting cracks in brittle solids: Further results. Int. J. Solids Structures 15, 111-126 (1979).

4. S. Nemat-Nasser and A. Oranratnachai, Minimum spacing of thermally induced cracks in brittle solids. $J$. Energy Resources Technology, Trans. ASME 101, 34-40 (1979).

5. S. Nemat-Nasser, Y. Sumi and L. M. Keer, Unstable growth of tension cracks in brittle solids: Stable and unstable bifurcations, snap-through and imperfection sensitivity. Earthquake Engineering and Research Laboratory, Tech. Rep. No. 79-7-20, Dept. Civil Engineering, Northwestern University, Evanston, Ill., July 1979; Int. J. Solids Structures 16, $1017-1035(1980)$.

6. S. Nemat-Nasser, Geothermal energy: Extracting heat from hot dry rock masses. Proc. 16th Midwestem Mech. Conf., Kansas State University, (19-21 Sept. 1979).

7. S. Nemat-Nasser and A. Oranratnachai, Minimum spacing of thermally induced cracks in brittle solids; Addendum. To appear.

8. A. Oranratnachai, S. Nemat-Nasser and L. M. Keer, Effect of geometric imperfection on stability of the growth regime of thermally induced interacting edge cracks. To appear.

\section{APPENDIX A}

The theory behind the stability chart shown in Fig. Al, taken from [7], will be summarized. The same dimensionless quantities as defined in Section 4 are used: dimensionless stress intensity factor $N$, dimensionless thermal length $\Delta$ and dimensionless crack length $a$. The assumed temperature profile due to heat conduction for these calculations is given by

$$
\begin{aligned}
T & =T_{0} \operatorname{erf}\left(\frac{y \sqrt{ } 3}{\delta}\right) \quad y \geq 0 \\
\operatorname{erf}(x) & =\frac{2}{\sqrt{ } \pi} \int_{0}^{x} \mathrm{e}^{-u^{2}} \mathrm{~d} u .
\end{aligned}
$$

For a given $\Delta$ and given temperature profile, the value of $N$ depends on $a$. The dashed curve OMP in Fig. Al is a plot of the maximum $N$ for a given $\Delta$ for this temperature profile. This curve therefore defines the unattainable region for $N$ : points above curve $O M P$ cannot be attained.

Consider two interacting cracks such that their dimensionless stress intensity factors are at the critical value, i.e. $N_{1}=N_{2}=N_{c}$. For an increment in the load parameter $\mathrm{d} \Delta>0$, the corresponding dimensionless crack lengths, $\mathrm{d} a_{1}$ and $\mathrm{d} a_{2}$, can be calculated such that the new state remains in equilibrium, i.e. $\mathrm{d} N_{1}=\mathrm{d} N_{2}=0$,

$$
\begin{aligned}
& \mathrm{d} N_{1}=\frac{\partial N_{1}}{\partial a_{1}} \mathrm{~d} a_{1}+\frac{\partial N_{1}}{\partial a_{2}} \mathrm{~d} a_{2}+\frac{\partial N_{1}}{\partial \Delta} \mathrm{d} \Delta=0, \\
& \mathrm{~d} N_{2}=\frac{\partial N_{2}}{\partial a_{1}} \mathrm{~d} a_{1}+\frac{\partial N_{2}}{\partial a_{2}} \mathrm{~d} a_{2}+\frac{\partial N_{2}}{\partial \Delta} \mathrm{d} \Delta=0
\end{aligned}
$$

From eqn (A2) it follows that

$$
\frac{\mathrm{d} a_{2}}{\mathrm{~d} a_{1}}=\left[\frac{\partial N_{2}}{\partial a_{1}} \frac{\partial N_{1}}{\partial \Delta}-\frac{\partial N_{1}}{\partial a_{1}} \frac{\partial N_{2}}{\partial \Delta}\right] /\left[\frac{\partial N_{1}}{\partial a_{2}} \frac{\partial N_{2}}{\partial \Delta}-\frac{\partial N_{2}}{\partial a_{2}} \frac{\partial N_{1}}{\partial \Delta}\right]
$$

Crack 2 is arrested when the numerator of this quotient ceases to be positive. Thus the critical state where the growth regime changes to one crack stopping as the other continues to grow, is characterized by

$$
\frac{\partial N_{2}}{\partial a_{1}} \frac{\partial N_{1}}{\partial \Delta}-\frac{\partial N_{1}}{\partial a_{1}} \frac{\partial N_{2}}{\partial \Delta}=0
$$




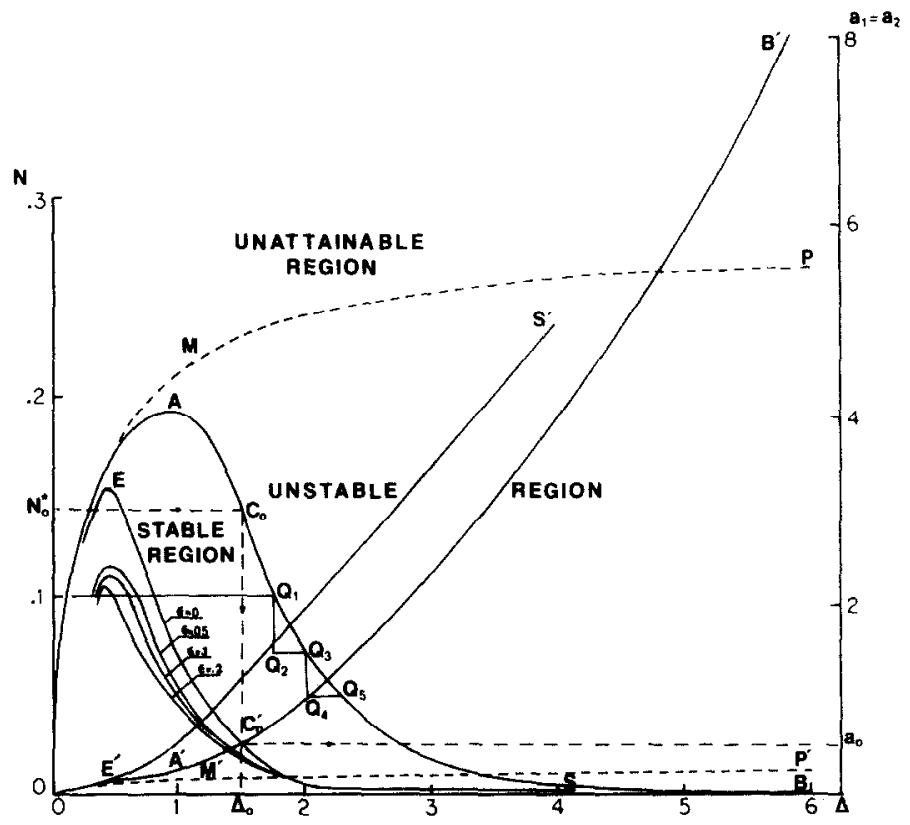

Fig. Al. Stability chart for temperature profile, eqn (A1).

By symmetry, for $a_{1}=a_{2}$,

$$
\frac{\partial N_{1}}{\partial \Delta}=\frac{\partial N_{2}}{\partial \Delta}, \frac{\partial N_{1}}{\partial a_{1}}=\frac{\partial N_{2}}{\partial a_{2}}, \frac{\partial N_{1}}{\partial a_{2}}=\frac{\partial N_{2}}{\partial a_{1}}
$$

Hence eqn (A4) reduces to

$$
\frac{\partial N_{1}}{\partial a_{1}}=\frac{\partial N_{2}}{\partial a_{2}}=\frac{\partial N_{1}}{\partial a_{2}}=\frac{\partial N_{2}}{\partial a_{1}}=0
$$

This condition defines the critical state for stable bifurcation of a perfect system, as defined in [5]. Two equilibrium states exist at this point, but the one with lower total stored elastic energy is preferred; this is the state with every other crack stopping as the remaining cracks continue to grow. In Fig. Al the curve OES corresponds to the loci of points for which eqn (A6) is satisfied. The stable attainable region is thus the portion below curve OES. The series of curves below OES corresponds to initial imperfections of 5,10 , or $20 \%$ in the dimensionless critical stress intensity factor. The results for initial imperfection in spacing are not the same as plotted here; see[8].

The curve $O A B$ is the loci of unstable bifurcation points. It corresponds to the condition

$$
\frac{\partial N_{1}}{\partial a_{1}}=\frac{\partial N_{2}}{\partial a_{2}}=0
$$

The solid curve $O E^{\prime} S^{\prime}$ in Fig. A1 denotes the values of $a_{1}=a_{2}$ corresponding to the value of $N_{1}=N_{2}$, for the same value of $\triangle$, on curve $O E S$. Curve $O A^{\prime} B^{\prime}$ similarly corresponds to $O A B$.

Figure $A 1$ can therefore be used to calculate the crack length and thermal depth at which every other crack stops. First the critical value of $N$ is calculated for the initial conditions. From curve $O E S$ the corresponding $\Delta$ is found, from which the value of $a$ is obtained using curve $O E^{\prime} S^{\prime}$. Repeating this procedure for the next value of $N$, calculated by dividing the previous value by $\sqrt{ } 2$, one arrives at the second level of crack interaction. The entire crack regime can thus be predicted by continuing this procedure.

Since the initial value of $N$ pertaining to our experiments, e.g. that corresponding to Fig. 5 , is small (e.g. $N=0.016$ ), it is seen from Fig. Al that material imperfection does not affect the estimate of critical values of $\Delta$ and $a$ appreciably. On the other hand geometric imperfections can become rather significant in the present case and 5-10\% imperfection in crack spacing can account for the difference in observed and estimated results; see [8]. 